Historic, Archive Document

Do not assume content reflects current scientific knowledge, policies, or practices. 


$$
\text { • }
$$




\section{GLAD BULBALOG}

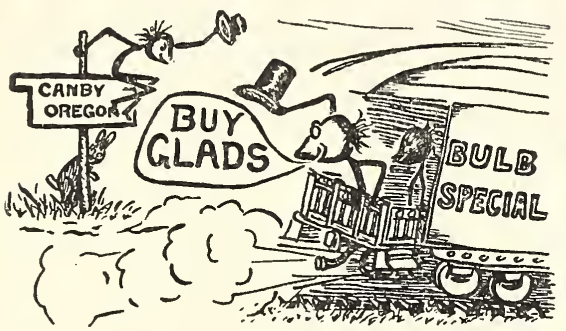

\section{Spring, 1931}

\section{Merton G. Ellis Bulb Gardens}

Our stock has been inspected under license No. 242

\section{Wholesale and Quantity}

Canlby, Dregén

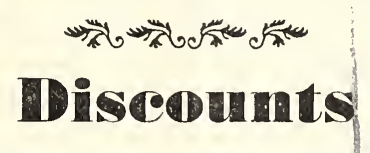

On all cash orders you may select extra Bulbs and Bulblets as follows:

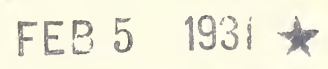

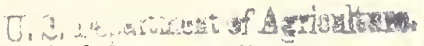

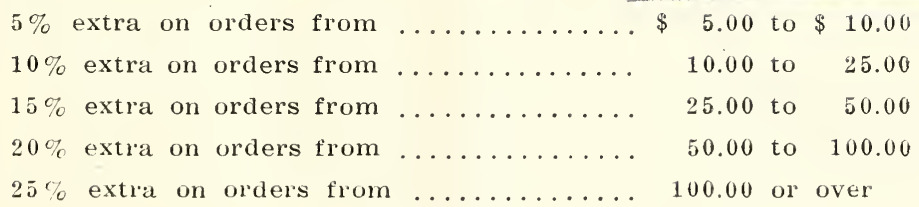

mएग्रण

\section{Safie Delivery Guaranteed by Wxpress}

We guarantee safe delivery by express on orders of $\$ 5.00$ or more, if you notify us and your express agent at once in case of damage by freezing or other loss or damage, so that we may file claim.

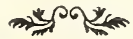 \\ Teroms}

Cash or $25 \%$ down and balance C. O. D. All orders are received subject to prior sale. Discounts do not apply on special quotations. 25 at fourth of 100 rate. Multiply 100 rate by 9 to get price per 1000 .

All who order 25 or more bulbs of a variety are entitled to order same from this list.

\section{CLOSED ON SUNDAYS •}

Priced F. O. B. Canby, Oregon 
GL.ADIOLUS

(For Descriptions see Retail List)

Angel's Dream

Allen V. Bunce

Annie Laurie

Beatricia

Beatriz Michelena

Bengal Tiger

Betty Joy

Bobby

Break o'Day

Byron L. Smith

Carmen Sylva

Catherine Coleman

Copper Bronze

Cowee's Scarlet Wonder

Crimson Glow

Diener's American Beauty

Dr. F. E. Bemnett

Elizabeth Tabor

Emile Aubrun

Ethelyn

Fern Kyle

Gertrude Errey

Gladdie Boy

Glendale

Gloriana

Gold Eagle

Golden Measure

Highland Laddie

Imp. Queen of the Night

J. A. Carbone

Jack London

Jacoba van Beijeren

Jenny Lind

John T. Pirie

Juniata

Kunderd's Yellow Wonder

Longfellow

Los Angeles
BULBS PER 100

No. 1 No. 2 No. $3 \quad$ No. 4 $\$ 75.00 \$ 65.00 \quad \$ 50.00 \quad \$ 36.00 \quad \$ 24.00 \quad \$ 18.00$

\begin{tabular}{|c|c|c|c|c|c|c|}
\hline & $\ldots . .$. & ...... & 2.00 & 1.35 & 1.00 & 1.50 \\
\hline & 6.00 & 5.00 & 4.00 & 3.00 & 2.00 & 2.00 \\
\hline 2.80 & 2.00 & 1.60 & 1.20 & .90 & .60 & .60 \\
\hline & ........ & 3.10 & 2.40 & 1.80 & .90 & $.8 j$ \\
\hline & 2.00 & 1.60 & 1.20 & .90 & .60 & .60 \\
\hline & $\ldots \ldots$. & ....... & 3.00 & 2.00 & 1.25 & 1.75 \\
\hline 4.50 & 3.80 & 3.10 & 2.40 & 1.80 & 1.20 & .80 \\
\hline 2.80 & 2.00 & 1.60 & 1.20 & .90 & .60 & .60 \\
\hline & 3.00 & 2.50 & 2.00 & 1.30 & .70 & .70 \\
\hline$\cdots$ & ....... & $\ldots .$. & 1.20 & .90 & .60 & .60 \\
\hline 6.80 & 5.80 & 4.80 & 3.80 & 2.80 & 1.4. & 1.30 \\
\hline 5.65 & 4.80 & 3.95 & 2.90 & 2.00 & 1.25 & .90 \\
\hline 2.80 & 2.00 & 1.60 & 1.20 & .90 & .60 & .60 \\
\hline & ...... & 1.60 & 1.20 & .90 & .60 & .60 \\
\hline …... & $\ldots \ldots$. & 1.60 & 1.20 & .90 & .60 & .60 \\
\hline 3.60 & 3.00 & 2.50 & 2.00 & 1.50 & .90 & 1.70 \\
\hline & 2.00 & 1.60 & $1.2 \mathrm{~J}$ & .90 & .60 & .60 \\
\hline & ...... & $\ldots \ldots$ & $\ldots \ldots$ & ....... & 2.75 & 2.00 \\
\hline 3.60 & 2.90 & 2.30 & 1.65 & 1.35 & .70 & $.7 v$ \\
\hline 2.80 & 2.00 & 1.60 & 1.20 & .90 & .60 & .60 \\
\hline . & $\ldots . .$. & 1.60 & 1.20 & .90 & .60 & .60 \\
\hline 4.50 & 3.80 & 3.10 & 2.40 & 1.80 & 1.20 & .80 \\
\hline & ....... & 1.60 & 1.20 & $.9 \mathrm{~J}$ & .60 & .60 \\
\hline 4.70 & 3.75 & 2.80 & 2.10 & 1.30 & .90 & .90 \\
\hline & 3.80 & 3.10 & 2.40 & 1.80 & 1.20 & .80 \\
\hline & 2.80 & 2.30 & 1.70 & 1.10 & .70 & .70 \\
\hline & $\ldots \ldots$ & $\ldots$ & 2.00 & 1.30 & .70 & .70 \\
\hline & ....... & 5.00 & 4.00 & 3.00 & 2.00 & 2.00 \\
\hline & $\ldots . .$. & 1.60 & 1.20 & .90 & .60 & .60 \\
\hline .... & 2.00 & $1: 60$ & 1.20 & .90 & .60 & .60 \\
\hline 3.60 & 2.80 & 2.30 & 1.70 & 1.10 & .70 & .70 \\
\hline .... & ....... & 1.60 & 1.20 & .90 & .60 & .60 \\
\hline & $\ldots$. & 2.30 & 1.70 & 1.10 & .70 & .70 \\
\hline 6.80 & 5.80 & 4.80 & 3.80 & 2.90 & 2.00 & 2.00 \\
\hline$\cdots$ & 3.80 & 3.10 & 2.40 & 1.80 & 1.20 & .80 \\
\hline 3.60 & 3.00 & 2.50 & 2.00 & 1.50 & .90 & .70 \\
\hline & 2.00 & $1.60 \%$ & 1.20 & .90 & .60 & .60 \\
\hline
\end{tabular}

Bulblets

No. 5 No. 6 Per 1000

$\$ 15.00$

60

8.

60

80

30

90

60

60

iv

00

;0

60

80

60

(1)

So

70

70

;0

70

60

70

00

80

70

60 
GLADIOLUS

(For Descriptions see Retail List)
BULBS PER 100

No. 1 No. 2 No. $3 \quad$ No. 4
Bulblets $\$ 2.80 \$ 2.00 \$ 1.60 \$ 1.20 \$ \quad .90 \$ \quad .60 \$ \$ \quad .60$ $\begin{array}{lllllll}3.60 & 2.80 & 2.30 & 1.70 & 1.10 & .70 & .70\end{array}$

Marietta

Marnia

Many Pickloul

Masterpiece

Minuet

Mr's. Dr. Norton

Mrs. F. C. Hormberger

Mrs. F. C. Peter's

Mrs. Leon Douglas

Mrs. P. W. Sisson

Mr. W. H. Phipls

Pink Lily

Pride of Purtand

l'ride of Wamakah

Princely

Red Fire

Richard Diener

Rose Ash

Star of the Sea

Srword of Mahomet

Tycko Zang

\begin{tabular}{|c|c|c|c|c|c|c|}
\hline & & & & & & \\
\hline ...... & ...... & 1.60 & 1.20 & .90 & .60 & .60 \\
\hline 2.80 & 2.00 & 1.60 & 1.20 & .90 & 60 & 60 \\
\hline ....... & 2.00 & 1.60 & 1.20 & .90 & .60 & .60 \\
\hline 2.00 & 10.00 & 8.50 & 7.00 & 5.00 & 3.00 & 7.00 \\
\hline ....... & ...... & ....... & $\ldots$ & .90 & .60 & .60 \\
\hline 6.80 & 5.80 & 4.80 & 3.80 & 2.90 & 2.00 & 2.00 \\
\hline ...... & 2.00 & 1.60 & 1.20 & .90 & .60 & .60 \\
\hline 2.80 & 2.00 & ...... & 1.20 & .90 & .60 & .60 \\
\hline ....... & ....... & 3.10 & 2.20 & 1.40 & .90. & .80 \\
\hline 4.50 & 3.80 & 2.90 & 2.00 & 1.25 & .75 & .80 \\
\hline ....... & 3.80 & 3.10 & 2.40 & 1.80 & 1.20 & .80 \\
\hline 60.00 & 50.00 & 40.00 & 30.00 & 20.00 & 10.00 & 10.00 \\
\hline ....... & ...... & ....... & ........ & 1.50 & 1.00 & 1.00 \\
\hline 2.80 & 2.00 & 1.60 & 1.20 & .90 & .60 & .60 \\
\hline 2.80 & 2.00 & 1.60 & 1.20 & .90 & .60 & .60 \\
\hline 2.80 & 2.00 & 1.60 & 1.20 & .90 & .60 & .60 \\
\hline$\ldots .$. & 2.00 & 1.60 & 1.20 & .90 & 60 & .60 \\
\hline 25.00 & 20.00 & 16.00 & 11.00 & 8.00 & 5.00 & 9.00 \\
\hline 9.00 & 7.50 & 6.00 & 4.50 & 3.00 & 2.00 & 3.00 \\
\hline ..... & $\cdots$ & ...... & $\cdots$ & 1.00 & .70 & .75 \\
\hline
\end{tabular}

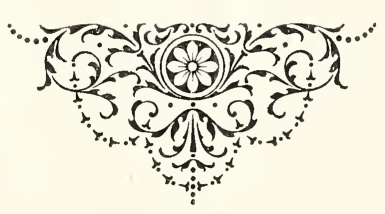

\section{Business Is Better}

Last fall and this spring our bulb sales have run much better than during the same period a year ago. Apparently the worst of the general business depression is over. Our increased sales may also be due in part to our

\section{High Quality Bulbs}

We grow the best varieties and use new soil each year. Our equipment is excellent for the handling of bulbs and we have just completed a large new addition to our already commodious bulb house. We have many fine new originations of our own which have also contributed largely to our success in winning the Grand Sweepstakes Gold Cup of the N. W. Regional Gladiolus Show for most points, the Sweepstakes Silver Cup for best spike, first for best general exhibit and first for best new seedling, all for three years straight. 


\section{读 Bulblets by the Quarot}

Priced F. O. B. Canby. Oregon

PINTS AT HALF OF QUART PRICES. WRITE FOR PRICES IN QUANTITY

\begin{tabular}{|c|c|c|c|}
\hline Angel's Dream ................. & 0.00 & K's Yellow Wonder & 1.75 \\
\hline Beatricia & 2.00 & Longfellow & 3.50 \\
\hline Bengal Tiger .................... & 1.50 & Los Angeles ...... & 1.50 \\
\hline Betty Joy & 6.75 & Marie Kunderd & 2.50 \\
\hline Bobby & 5.00 & Marietta & 3.00 \\
\hline Break o'Day .... & 1.25 & Marshal Foch ... & 2.00 \\
\hline Carmen Sylva & 1.50 & Mary Pickford & 1.25 \\
\hline C's Scarlet Wonder .... & 1.25 & Masterpiece. & 250 \\
\hline Crimson Glow ............... & 1.50 & Minuet & 36.00 \\
\hline Dr. F. E. Bennett & 4.50 & Mrs. Dr. Norton & 1.25 \\
\hline D's Am. Beauty ...... & 150 & Mrs. F. C. Peters & 1.50 \\
\hline Elizabeth Tabor & 1.25 & Mrs. F. C. Hornberger & 4.50 \\
\hline Ethelyn ...... & 2.50 & Mrs. Leon Douglas & 2.00 \\
\hline Fern Kyle ........ & 2.50 & Mrs. P. W. Sisson & 3.50 \\
\hline Gertrude Errey & 1.50 & Mr. W. H. Phipps.. & 3.50 \\
\hline Gladis Plath & 1.75 & Pink Lily & 3.00 \\
\hline Gladdie Boy .... & 2.50 & Princely & 1.50 \\
\hline Glendale ........... & 2.00 & Prince of Wales & 3.00 \\
\hline Gloriana .......................... & 4.50 & Red Fire ...... & 2.00 \\
\hline Gold Eagle ........................ & 2.50 & Richard Diener & 2.50 \\
\hline $\begin{array}{l}\text { Golden Measure } \\
\text { Halcyon }\end{array}$ & $\begin{array}{l}2.50 \\
5.00\end{array}$ & $\begin{array}{l}\text { Richard Diener .... } \\
\text { Robt. J. Kunderd }\end{array}$ & 3.50 \\
\hline Imp. Queen of Night & 5.00 & Rose Ash ................... & 1.25 \\
\hline J. A. Carbone ................ & 2.00 & Scarlet Wonder & 1.25 \\
\hline Jack London ............... & 1.50 & Sword of Mahomet .... & 12.00 \\
\hline Jenny Lind & 2.00 & Star of the Sea & 45.00 \\
\hline John D. Oliver .............. & 4.00 & rycko Zang ....................... & 2.85 \\
\hline John T. Pirie & 2.00 & Uhlan Chief & 4.50 \\
\hline Juniata ............. & 6.00 & Veiled Brilliance & 10.00 \\
\hline
\end{tabular}

BANK REFERENCE: THE CANBY STATE BANK, CANBY, OREGON

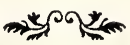

\section{Merton G. Ellis Bulb Gardens Canby, Oregon}

